\title{
Propagation dynamics of ultrashort pulses in nonlinear fiber couplers
}

\author{
Nail Akhmediev \\ Optical Sciences Centre, Institute of Advanced Studies, The Australian National University, \\ Canberra, Australian Capital Territory 2601, Australia \\ J. M. Soto-Crespo \\ Instituto de Óptica, Consejo Superior de Investigaciones Científicas, Serrano 121, 28006 Madrid, Spain
}

(Received 31 August 1993)

\begin{abstract}
The nonlinear fiber coupler is considered as a Hamiltonian dynamical system with an infinite number of degrees of freedom, with the soliton states of the coupler being the singuiar points of this dynamical system. Numerical simulations show that arbitrary initial conditions give rise, asymptotically, to oscillations around some of the stable singular points and some amount of radiation. Examples of different initial conditions, including unstable soliton states and single pulses launched in one channel of the coupler are considered.
\end{abstract}

PACS number(s): 42.81.Dp, 42.65.Pc, 52.35.Mw

\section{INTRODUCTION}

The reason for the current increase of interest in all optical switching devices is that these devices can potentially operate at speeds much higher than those possible with electronic or optoelectronic switches. The nonlinear directional coupler has certainly been the most frequently studied device since it was proposed [1,2]. Switching properties of this device, using continuous wave (cw) and very short pulses, have been extensively studied both theoretically [3-16] and experimentally [17-20]. It has been shown experimentally [20] that input pulses as short as $100 \mathrm{fsec}$ can be used to obtain complete switching. In principle, this is not the fastest switching time that can be achieved with directional couplers.

Although much work has already been done to understand the processes taking place in a nonlinear fiber coupler, the theory of this device is still incomplete. Only special (and simple) cases have been considered using numerical $[5-7,9]$ or semianalytical $[8,10,12-15]$ approaches. On the other hand, experimental data have shown that pulse propagation on this device is not always so simple. For example, the pulse can break up into smaller pulses [18]; this effect, which leads to incomplete self-switching, cannot be explained using simple theories. Hence, a more elaborate approach is needed. The aim of this paper is to develop a general approach which can answer the question: what will happen if we excite a nonlinear directional coupler with an arbitrary pair of pulses? In principle, this kind of general approach can help to develop the theory of any particular device which is based on the nonlinear coupler, e.g., the optical transistor [1], pulse compressors [21], logic elements [22], etc.

From a mathematical point of view the problem of wave propagation in a nonlinear fiber coupler reduces to a study of the properties of the solutions of a pair of coupled nonlinear Schrödinger equations (NLSE's) with a linear coupling term [5]. This set of equations is nonintegrable in the sense that methods such as the inverse scattering technique cannot be applied to it. Therefore, the initial value problem cannot be solved analytically. Nevertheless, two integrals - the energy invariant and the Hamiltonian of the set-exist and the corresponding dynamical system with an infinite number of degrees of freedom can be considered as Hamiltonian. This means that some general methods of the theory of Hamiltonian dynamical systems can be applied to the set, and a systematic investigation of its solutions can be made. Although the theory of dynamical systems has been developed mostly for finite dimensional systems described by ordinary differential equations $[23,24]$, many features of it can be applied to infinite-dimensional systems described by partial differential equations [25].

The most characteristic feature of a dynamical system described by nonlinear evolution equations is that smooth bound initial conditions give rise, during propagation, to a finite number of bound states (solitons) plus some amount of radiation, which disperses in time (or diffracts in space). The classical example of this type of behavior is the standard NLSE [27] with bound initial conditions. This happens in more complicated cases of systems which are Hamiltonian but nonintegrable [28]. Menyuk recently pointed out [26] that solitons of integrable systems are robust in the presence of Hamiltonian deformations. In fact, even if the dynamical system is far from integrable but is still Hamiltonian, it can admit soliton states which in some aspects behave similarly to those in integrable systems.

Our numerical simulations show that this is the case for the nonlinear directional couplers studied in this paper. In contrast to simple systems described by a single NLSE, the coupled set of NLSE's has composite soliton states rather than single solitons. Moreover, the coupled set of NLSE's has several types of soliton states [29], although not all of them are stable [30]. Hence, we expect that asymptotically, the output of the coupler will consist of a finite number of stable soliton states and a certain amount of dispersed radiation. In contrast to solitons in integrable systems, stable soliton states of the coupler are usually excited with oscillatory perturbations. 
From this point of view, the systematic investigation of a Hamiltonian dynamical system should consist of the following steps (either analytically or numerically): (i) Finding the stationary solutions (singular points); (ii) investigation of their stability (establishing the type of point); (iii) study of the evolution of the unstable states; and (iv) solution of the initial value problem. Step (i) of this sequence has been done previously in Ref. [29], where the full set of soliton states for the coupled set of nonlinear Schrödinger equations has been found and a bifurcation diagram for them has been constructed. Step (ii) has been partly done (for symmetric and antisymmetric states in a limited range of parameters) in Refs. [6] and [13]. A comprehensive study of the stability properties of the full set of soliton states has been recently completed in [30]. Step (iii), and partially step (iv), are accomplished in this work. Numerical simulations starting from unstable symmetric and antisymmetric states, which are also in agreement with our general conclusion, have been done previously in Ref. [6].

The results from steps (iii) and (iv) can be found asymptotically for large distances of propagation, where the soliton states are well separated from the radiation and from each other. This is analogous to a single NLSE where asymptotic results can be predicted from the spectrum of the inverse scattering problem. At short propagation distances there is still a strong interaction between the different parts of the solution, and so each case has to be considered separately. Both cases, i.e., short and long couplers, can be interesting in practice. Note also that we are considering here only the cases when two or more soliton states are well separated at the output. The cases when they are superimposed on each other are more complicated and require more numerical simulations to understand the processes in the coupler.

The remainder of this paper is organized as follows. In Sec. II we formulate the problem, summarizing the main conclusions obtained in Refs. [29] and [30]. In Sec. III we present some numerical results on the evolution of the unstable states. In Sec. IV we present several numerical results on the initial value problem. In Sec. V we summarize and discuss our results, comparing them with previous ones from other authors. Finally, Sec. VI contains our conclusions.

\section{SOLITON STATES OF A NONLINEAR COUPLER}

Pulse propagation in a dual-core fiber coupler, including the effects of dispersion to second order and selfphase modulation can be described in terms of two linearly coupled nonlinear Schrödinger equations. In a reference frame traveling along the $\xi$ axis with the light group velocity, this set of equations takes the form [5]

$$
\begin{aligned}
& i U_{\xi}+\frac{1}{2} U_{\tau \tau}+|U|^{2} U+K V=0, \\
& i V_{\xi}+\frac{1}{2} V_{\tau \tau}+|V|^{2} V+K U=0,
\end{aligned}
$$

where $U(\xi, \tau)$ and $V(\xi, \tau)$ are the electric field envelopes, $K$ is the normalized coupling coefficient between the two cores, $\xi$ is the normalized longitudinal coordinate, $\tau$ is the normalized retarded time, and the equations are writ- ten assuming anomalous group-velocity dispersion (GVD).

An invariant of Eqs. (1) is the Hamiltonian,

$$
\begin{gathered}
H=\int_{-\infty}^{\infty}\left[\frac{\left|U_{\tau}\right|^{2}-\left|V_{\tau}\right|^{2}}{2}-\frac{|U|^{4}+|V|^{4}}{2}\right. \\
\left.-K\left(U V^{*}+U^{*} V\right)\right] d \tau,
\end{gathered}
$$

where an asterisk means complex conjugate. Using Eq. (2), Eqs. (1) can be written in a canonical form

$$
i U_{\xi}=\frac{\delta H}{\delta U^{*}}, i V_{\xi}=\frac{\delta H}{\delta V^{*}} .
$$

Equations (2) and (3) define the Hamiltonian dynamical system on an infinite-dimensional phase space of two complex functions $U, V$ decreasing to zero at infinity. We assume that the functions $U$ and $V$ are smooth functions of both variables.

Separating explicitly the fast oscillatory part $e^{i q \xi}$ from the envelope functions, we can represent the solutions of Eq. (1) in the form

$$
\begin{aligned}
& U(\xi, \tau)=u^{\prime}(\xi, \tau) e^{i q \xi}, \\
& V(\xi, \tau)=v^{\prime}(\xi, \tau) e^{i q \xi},
\end{aligned}
$$

where $q$ is the spatial frequency shift and $u^{\prime}(\xi, \tau)$ and $v^{\prime}(\xi, \tau)$ are the new envelope functions. It is supposed that these functions do not contain fast oscillations. The value $q$ can be considered as the parameter of a soliton state family of solutions. It is analogous to the propagation constant in the theory of nonlinear guided waves [31]. The rest of the parameters of the soliton states depend on $q$. By inserting Eqs. (4) into Eqs. (1), one obtains

$$
\begin{aligned}
& i u_{\xi}^{\prime}+\frac{1}{2} u_{\tau \tau}^{\prime}-q u^{\prime}+\left|u^{\prime}\right|^{2} u^{\prime}+K v^{\prime}=0 \\
& i v_{\xi}^{\prime}+\frac{1}{2} v_{\tau \tau}^{\prime}-q v^{\prime}+\left|v^{\prime}\right|^{2} v^{\prime}+K u^{\prime}=0
\end{aligned}
$$

which has two parameters $q$ and $K$. Using the following rescaling,

$$
u^{\prime}=u \sqrt{K}, \quad v^{\prime}=v \sqrt{K}, \quad t=\tau \sqrt{K}, \quad z=K \xi,
$$

Eqs. (5) become

$$
\begin{aligned}
& i u_{z}+\frac{1}{2} u_{t t}-\frac{q}{K} u+|u|^{2} u+v=0, \\
& i v_{z}+\frac{1}{2} v_{t t}-\frac{q}{K} v+|v|^{2} v+u=0,
\end{aligned}
$$

so that there is now only one combined parameter, viz., $q / K$. The latter transformation shows that all results can be presented in terms of this combined parameter.

Equations (1) have a second invariant:

$$
\begin{aligned}
Q=\int_{-\infty}^{\infty}\left(|U|^{2}+|V|^{2}\right) d \tau & =\int_{-\infty}^{\infty}\left(\left|u^{\prime}\right|^{2}+\left|v^{\prime}\right|^{2}\right) d \tau \\
& =\sqrt{K} \int_{-\infty}^{\infty}\left(|u|^{2}+|v|^{2}\right) d t
\end{aligned}
$$

which corresponds to the total energy transmitted by a 
signal in the fiber coupler.

A useful integral relation is

$\frac{d(\Delta Q)}{d \xi}=4 i \operatorname{Im} \int_{-\infty}^{\infty} U V^{*} d \tau=4 i \sqrt{K} \operatorname{Im} \int_{-\infty}^{\infty} u v^{*} d t$,

where $\quad \Delta Q=\int_{-\infty}^{\infty}\left(|U|^{2}-|V|^{2}\right) d \tau=\sqrt{K} \int_{-\infty}^{\infty}\left(|u|^{2}\right.$ $\left.-|v|^{2}\right) d t$ is the difference between the energies carried by each core. Equation (9) shows that the rate of energy exchange between cores depends on the relative phase of their field envelopes.

Equations (5) have stationary (independent on $\xi$ ) solutions: $u_{0}^{\prime}(\tau), v_{0}^{\prime}(\tau)$. These solutions can be found by solving Eqs. (5), with the terms involving the derivatives with respect to $\xi$ set to zero. In particular, the set of equations (5) has symmetric,

$$
u_{0}^{\prime}=v_{0}^{\prime}=\frac{\sqrt{2(q-K)}}{\cosh [\sqrt{2(q-K)} \tau]},
$$

and antisymmetric,

$$
u_{0}^{\prime}=-v_{0}^{\prime}=\frac{\sqrt{2(q+K)}}{\cosh [\sqrt{2(q+K)} \tau]},
$$

solutions.

In addition to symmetric and antisymmetric states, the set of equations (5) has also asymmetric solutions with unequal field components $u_{0}^{\prime}$ and $v_{0}^{\prime}$. There are no analytic solutions for them, but they can be found numerically (see Ref. [29]). There are two different families of asymmetric solutions; they bifurcate from the symmetric and antisymmetric states at $q / K=\frac{5}{3}$ and $q / K=1$, respectively. They have been named in Ref. [29]: $A$ - and $B$-type asymmetric states. Figure 1, taken from Ref. [29], shows the dispersion curves on the $(Q, q)$ plane for symmetric, antisymmetric, and asymmetric sohton states. It is seen from Fig. 1(a) that the $A$ - and $B$-type asymmetric states split off from the symmetric and antisymmetric states at bifurcation points $M$ and $N$ respectively.

An exhaustive study of the stability of all of these types of soliton states can be found in Ref. [30]. Here we

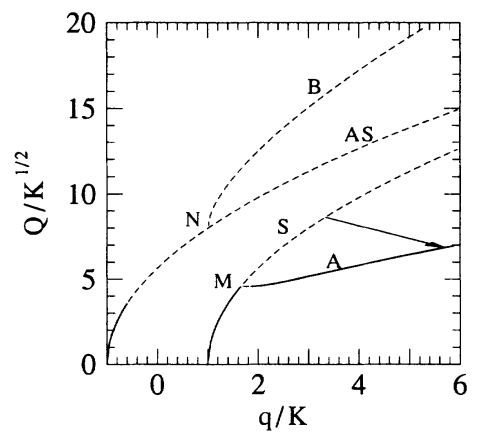

FIG. 1. Total energy carried by the soliton states vs the normalized soliton parameter $q$. Solid lines correspond to stable branches and dashed lines to unstable branches. The arrow shows an example of the transformation of an unstable symmetric state into a stable asymmetric $A$-type state. Note that the curves for the symmetric $(S)$ and $A$-type asymmetric $(A)$ soliton states have stable as well as unstable branches. briefly summarize the results of [30], as they are needed later in this paper.

(i) The symmetric soliton states are stable up to the point of bifurcation. They are unstable beyond the point of bifurcation and it is in this range of $q$ values that asymmetric $A$-type soliton states exist. In the region of instability there is only one (even) perturbation function with a real growth rate. This is an example of a symmetrybreaking instability [6].

(ii) For the antisymmetric soliton states there are even and odd perturbation functions which give instability. The odd perturbation function has the larger growth rate, and the eigenvalue can be complex for certain values of $q$. As a result, the antisymmetric states are unstable in almost the whole range of $q$ values where they exist. The range of $q$ values where even eigenfunctions exist is small compared to the range where odd perturbation functions exist. The growth rate values for them are lower than those for odd functions. Hence, their influence on the field evolution in nonlinear couplers will be less pronounced.

(iii) The asymmetric $A$-type soliton states are unstable at the values of $q$ where $d Q / d q$ is negative. The only eigenfunction of perturbation in this region is even. The $A$-type states are stable in the part of the dispersion curve where $d Q / d q$ is positive.

(iv) In the case of asymmetric $B$-type states, there are two different (even and odd) bound perturbation functions. The growth rates for both of them are real in the entire region where $B$-type soliton states exist. As both eigenvalues are real, these states are all unstable. The two eigenvalues for even and odd perturbation functions are of the same order of magnitude. This means that the evolution of unstable $B$-type states can be quite complicated in general. For this reason, in the following sections we will present numerical results for evolution of all unstable states except $B$-type states. The study of the evolution of these states deserves special investigation.

The above results concerning the stability of the different type of soliton states are summarized in Fig. 1. The continuous curves denote the stable branches and the dashed curves stand for those found to be unstable.

\section{EVOLUTION OF THE UNSTABLE SOLITON STATES}

Figure 1 indicates that if we launch into the coupler a symmetric soliton state with lower energy than that corresponding to the bifurcation point $M$, it will propagate without changing its profile at all. The same would happen with an asymmetric $A$-type soliton state in the region $d Q / d q \geq 0$. In this section we shall consider a few examples of the evolution of the unstable soliton states. We cannot cover all possible cases, of course, but some general conclusions can be drawn from these examples. It is shown that unstable soliton states can be transformed into the stable soliton states of another branch. The stable states belong to the elliptic type of singular points (or centers) for our infinite dimensional dynamical system, and the unstable soliton states are saddle-type points. In principle, elliptic type singular point cannot be 
reached by any trajectory. The presence of radiation can partly transform the elliptic type points into stable foci or limit cycles. Hence, the radiation of energy from the signal plays an important role in the transition from a saddle-type singular point into an elliptic type point.

We have solved Eq. (1) numerically by using a splitstep Fourier method on a time interval $\left[-\tau_{\max }, \tau_{\max }\right]$, where $\tau_{\max }$ is much bigger than the width of the initial input pulses. The temporal grid size was chosen to have up to 8192 points (usually 2048). The step size in the $\xi$ direction was typically taken to be $\Delta \xi=0.001$. Numerical accuracy was checked by repeating the simulations for different grid and step sizes. The calculations were performed using the following initial conditions:

$$
\begin{aligned}
& U(0, \tau)=u_{0}^{\prime}(\tau)+\mu f(\tau), \\
& V(0, \tau)=v_{0}^{\prime}(\tau)+\mu h(\tau),
\end{aligned}
$$

where $u_{0}^{\prime}(\tau)$ and $v_{0}^{\prime}(\tau)$ are some unstable soliton state, $f(\tau)$ and $h(\tau)$ are the perturbation functions as obtained in Ref. [30], and $\mu$ is a small parameter.

In the first example we chose a coupler with $K=0.3$, and as initial condition we took the symmetric stationary state associated with $q=1$, to which we added the corresponding eigenperturbations multiplied by $\mu=0.001$. Any other situation, with different $q$ and $K$ but the same $q / K$, produces identical results. Figure 2 shows the initial stationary state $u_{0}^{\prime}\left(=v_{0}^{\prime}\right)$ and its corresponding perturbative function $f$, which is equal to $-h$.

Figures 3(a) and 3(b) show the pulse profiles at different propagation distances $\xi$, for the above input. We can see that the pulses keep their even shape and that several small sidepulses split off from the central part. This central part can be considered as the signal. The side pulses are emitted from the signal, partly reducing its energy. The emission of these small subpulses from the signal can be considered as radiation which mostly propagates away from the signal and spreads out. Radiation emission is a general and unavoidable property of this problem. Although the dynamical system is Hamiltonian, the radiation emission makes the signal lossy. This could change the properties of the central pulse in propagation. We used some absorbers at the edges of the numerical grid to prevent the reflection of the radiation back to the center.

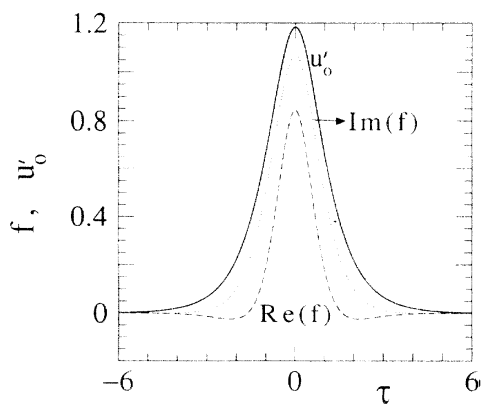

FIG. 2. Real (dashed line) and imaginary (dotted line) parts of the eigenperturbation $(f=-g)$ of the symmetric state at the point $q / K=0.333$. The shape of the initially symmetric state $u_{0}^{\prime}=v_{0}^{\prime}$ is shown by a solid line.
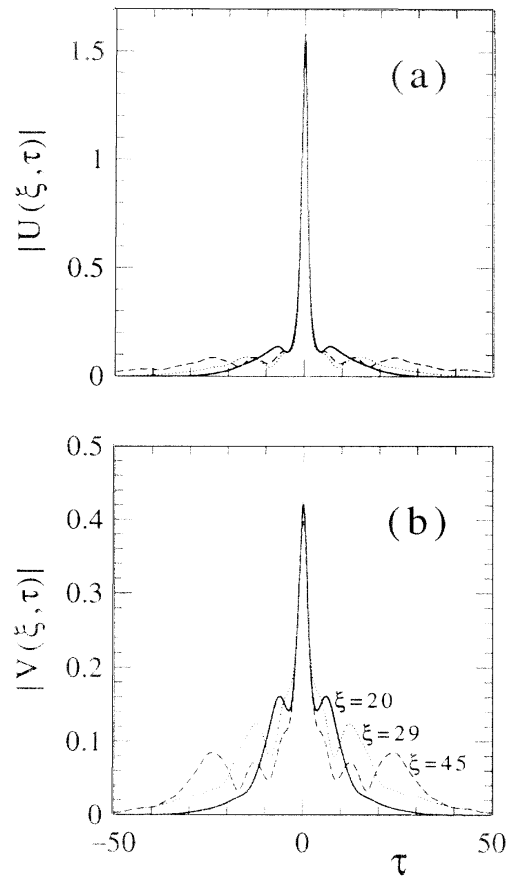

FIG. 3. Pulse profiles (a) $|U|$ and (b) $|\boldsymbol{V}|$ upon instability transformation at the propagation distances $\xi=20$ (continuous line), $\xi=29$ (dotted line), and $\xi=42$ (dashed line).

Note the asymmetry between the $U$ and $V$ functions and the different scales in the $y$ axes of Figs. 3(a) and 3(b).

The evolution of the peak amplitude of both pulses $|U(\tau=0)|$ and $|V(\tau=0)|$ is shown in Fig. 4(a). Because $f=-h$ the pulse amplitudes change in opposite ways. After propagating a certain distance up to $\xi=3$, during which the pulses hardly change, the field amplitudes diverge exponentially from their stationary values and after a short distance reach a different mean value, around which they oscillate. This oscillatory behavior is presented in Fig. 4(b), where we have plotted the imaginary vs the real part of the functions $Y_{1}=U(\xi, \tau=0) \exp (-i 1.71 \xi) \quad$ and $\quad Y_{2}=V(\xi, \tau=$ $0) \exp (-i 1.71 \xi)$, where $\xi$ is the parameter of these curves. Two initially close trajectories separate exponentially and rotate around two different fixed points.

These two fixed points are obviously centers (or elliptic points) according to the theory of dynamical systems [24]. The presence of radiation makes them behave more like stable foci or limit cycles. The oscillations occur around certain points with distances $U_{0}$ and $V_{0}$ from the origin; these can be determined exactly. Note the exponential factor $\exp (-i 1.71 \xi)$, which makes these points motionless. Effectively, this factor corresponds to a shift in the spatial frequency of the signal. That is, the $q$ value of the signal, as well as its energy, is shifted on propagation. The final state is obviously the asymmetric $A$-type state (or oscillations around it) associated with $q=1.71$. We have checked that the whole shape of this solution oscillates around the profile of this $A$-type state. This simulation is an example of a transformation from an unstable symmetric state into an asymmetric $A$-type state with $q / K=5$.7. This transformation is indicated in Fig. 


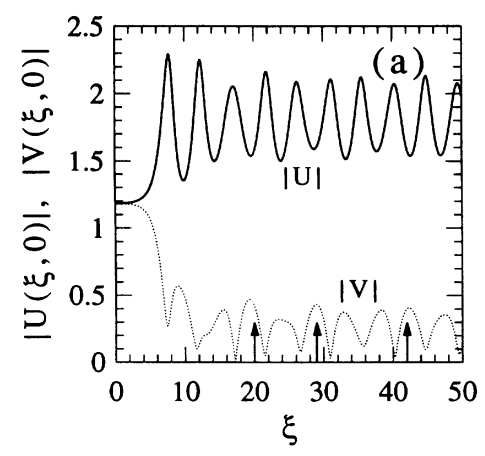

coupler, could be more reliable than those suggested before. On the other hand, this effect could be used for a precise comparison of the amplitudes of two pulses by launching them into the two inputs of the coupler.

Because of the stability of the asymmetric $A$-type soliton state, the convergence to this state is not direct but involves an oscillatory approach. This means that the final state can be a limit cycle rather than the exact asymmetric state. Nevertheless, this limit cycle is located around the $\boldsymbol{A}$-type state and can be described in an approximate form by

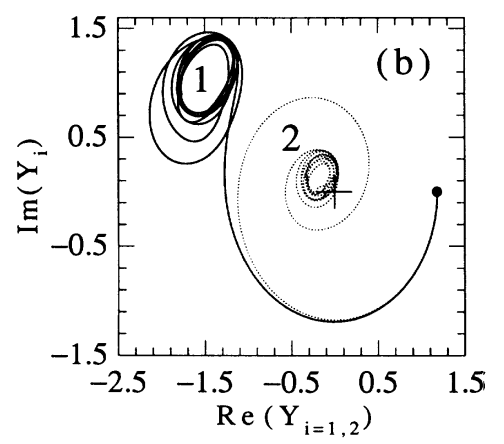

$$
\begin{aligned}
u^{\prime}(\xi, \tau)= & u_{0}^{\prime}(q, \tau)+\operatorname{Re}[f(q, \tau)] \cos (\omega \xi) \\
& +i \operatorname{Im}[f(q, \tau)] \sin (\omega \xi) \\
v^{\prime}(\xi, \tau)= & v_{0}^{\prime}(q, \tau)+\operatorname{Re}[h(q, \tau)] \cos (\omega \xi) \\
& +i \operatorname{Im}[h(q, \tau)] \sin (\omega \xi)
\end{aligned}
$$

where $\omega$ is defined by $\delta=i \omega, \delta$ being the purely imaginary eigenvalue associated with the perturbation $(f, h)$. For the $A$-type soliton states, the frequency $\omega$ of its single eigenfunction depends on its $q$ value.

This type of behavior of the output pulses is rather general. Arbitrary initial conditions give rise to some of the stationary solutions and additional radiation emitted from the main pulses, depending on the initial energy. Concerning the antisymmetric states, Fig. 5 clearly shows that the energy of any unstable antisymmetric state is sufficient to excite two other soliton states. The heavy solid line in this figure shows the energy of the antisymmetric state divided by 2 . It crosses the point $M$ and is higher than each of the stable branches of the symmetric and asymmetric $\boldsymbol{A}$-type states. Hence, an antisymmetric state could evolve into two symmetric or two asymmetric $A$-type states upon propagation.

We have studied the evolution of many antisymmetric states with initial conditions (12). For these states it is shown in Ref. [30] that the eigenperturbation with the largest growth rate $(f, h)$ satisfies $f=h$, and that it is an odd function of $\tau$. Figure 6 illustrates an antisymmetric stationary solution, the one associated with $q=1$ and $K=0.85$, and its corresponding eigenperturbation $f$. The perturbation functions for any other $q / K$ qualitatively have the same shape. Obviously, the odd perturbation

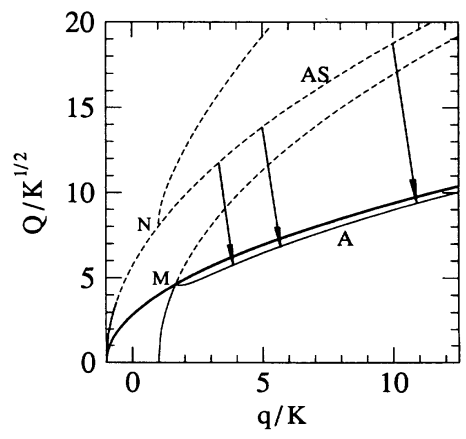

FIG. 5. The same as in Fig. 1. The heavy solid line shows the energy of the antisymmetric states divided by 2 . The arrows indicate the transformations of antisymmetric states into two $A$ type asymmetric states. 


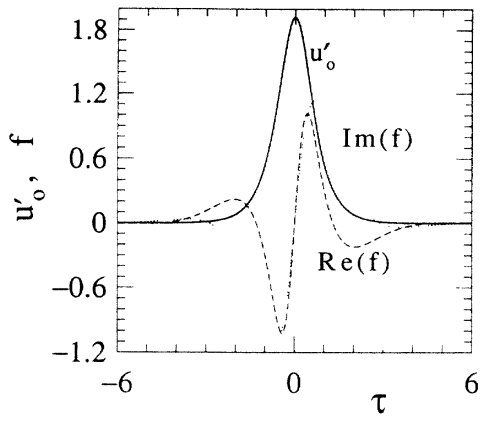

FIG. 6. Real (dotted line) and imaginary (dashed line) parts of the perturbation $[f(\tau)=g(\tau)]$ for the antisymmetric state at the point $q / K \approx 1.176$. The shape of the antisymmetric state $u_{0}^{\prime}=-v_{0}^{\prime}$ is shown by a solid line for comparison.

function will make the initially even functions $|U|$ and $|V|$ asymmetric on propagation.

Some examples of the evolution of these antisymmetric states are shown in Figs. 7(a) and 7(b), where the inputs correspond to $q / K=3.33$ and 10 . These inputs were launched in couplers with $K=0.3$ and 0.1 , respectively. Because of the conditions $f=h$ and $u_{0}^{\prime}=-v_{0}^{\prime}$, the pulses in both channels evolve keeping the symmetry $|U(\tau)|=|V(-\tau)|$, for all $\xi$. For this reason only one of the functions $(|U|)$ is shown in these figures. The other one $(|V|)$ is just the mirror image of $|U|$.

As can be seen from these figures, the antisymmetric states keep their shapes for some propagation distance, which depends on $\mu$, and then split off into two $A$-type asymmetric soliton states. Using the method of the trajectories in the complex plane, we have found that these
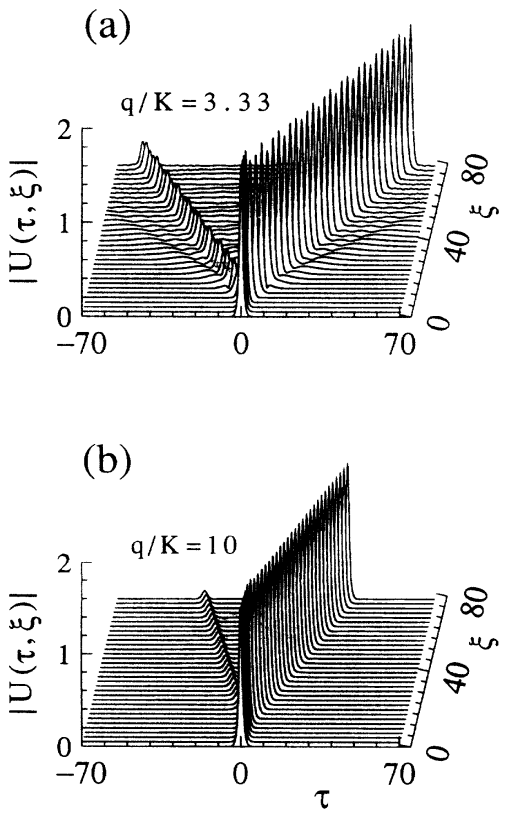

FIG. 7. Two examples of transformations of antisymmetric states into $\boldsymbol{A}$-type asymmetric states. In both cases the calculations were done up to $\xi=80$. The initial values of $q / K$ are written above the curves. two soliton states are two stable $A$-type soliton states with $q / K$ values of 3.9 and 10.9 , respectively. These processes are indicated in Fig. 5 by the arrows. The left and right arrows correspond to the above simulations. The arrow in the middle is one additional example of such transformation. The initial energy is more than the sum of the energy of the two final $A$-type solitons states. Again, the surplus energy is radiated in the form of small subpulses. The radiation is clearly seen in Fig. 7(a) but becomes negligible in the case of Fig. 7(b).

The two asymmetric $A$-type states separate from each other. This means that two $A$-type states will appear at the output with certain delay, which depends on the length of the coupler. This delay at the output can be much larger than the width of the pulses and for a given coupler depends on the $q$ value of the initially antisymmetric state. The sequence of appearance of the two asymmetric $A$-type states is defined by the sign of the perturbation in the initial conditions (12). Mathematically speaking, the small odd perturbations on the top of the even functions are equivalent to two even pulses shifted slightly in time. For small perturbations this initial shift is much smaller than the width of the pulses itself. These types of initial conditions have been used in the numerical simulations of Refs. [6] and [9] [see Figs. 5-8 of [6] and Fig. 6(a) of [9]] where results similar to our Fig. 7 were obtained. From a practical point of view, using initial conditions in the form of two pulses with opposite signs and with a small delay between them allows us to switch the sequence of the two soliton states at the output just by changing the sign of the small delay.

Using our approach, we obtain a complete understanding of the processes occurring when initial conditions close to the antisymmetric soliton states are used. If we decrease the total energy of the initial antisymmetric state shifting to the left along the curve $A S$ in Fig. 1 $(-0.6<q / K<0.71)$ then the perturbation to the antisymmetric states has complex eigenvalues with a comparatively small real part. This means that the instability becomes weaker and that it involves oscillations on propagation. If we further decrease the energy of the soliton states $(q / K<-0.6)$ then the antisymmetric states become stable.

As a third case, we used initial conditions in the form of the asymmetric $A$-type soliton state at $q / K=1.7$, the coupler having $K=0.59$. The $A$-type state is unstable in this region with a comparatively low value of the growth rate (see Ref. [30]). The pulse behavior is slightly different from the two previous cases due to the proximity of two stable branches: the symmetric and the $A$-type asymmetric states. The trajectories become periodic without any noticeable radiation. In fact, starting from the $A$-type unstable state, we can obtain periodic motion around either of the stable states, depending on the sign of the perturbation. These two types of trajectories are shown in Figs. 8(a) and 8(b). There is almost no radiation in this case and the orbit remains oscillatory during the whole evolution process. The $q$ value changes during this transformation. In Fig. 8(a) the corresponding phase factor is $\exp (-i 1.068 \xi)$ and in Fig. $8(\mathrm{~b})$ it is $\exp (-i 0.989 \xi)$. The former value of $q$ corresponds to 

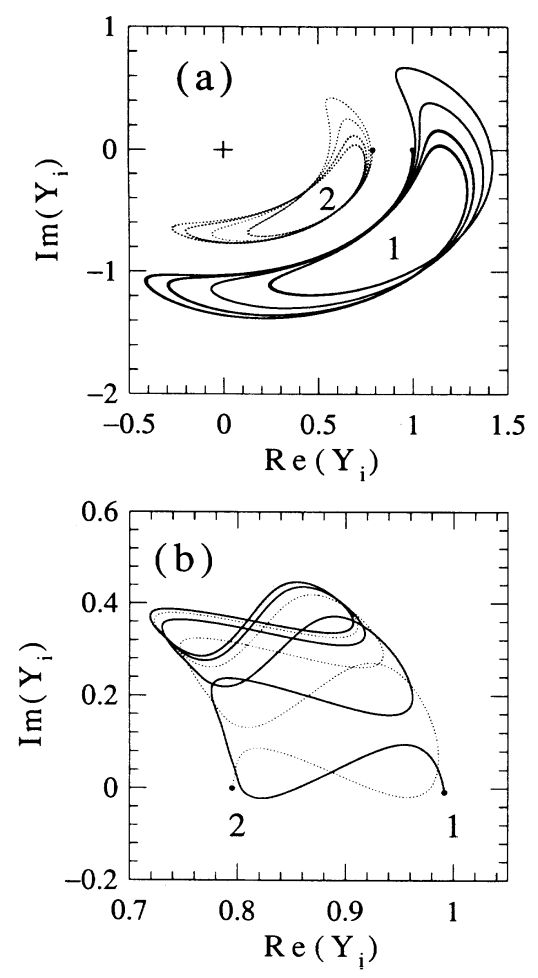

FIG. 8. Evolution of the imaginary vs the real part of the field at $\tau=0$ of an unstable $A$-type soliton state. (a) Negative perturbation: $\mu=-0.01$ in Eq. (12). The data are presented in a frame which includes the additional phase factor $\exp (-i 1.068 \xi)$. The filled circle is the starting point of the simulation $(\xi=0)$. (b) Positive perturbation: $\mu=0.01$. The data are presented in a frame which includes the additional phase factor $\exp (-i 0.989 \xi)$. Trajectory 1 in both cases corresponds to the function $U(\xi, \tau=0)$ and trajectory 2 to the function $V(\xi, \tau=0)$.

the $A$-type stationary state at the same energy level as the initial soliton state, that is $q=1$. The latter value corresponds to the symmetric state at the same energy level. Trajectories 1 and 2 are associated with $U$ and $V$, respectively, multiplied by the above phase factors, as in Fig. 3(b). The oscillations of these two trajectories around two different points in the complex plane show that the limiting case is an asymmetric $A$-type state. The trajectories are gradually converging to the center. The oscillations of both trajectories in Fig. 8(b) take place around the same point, which shows that the central point is a symmetric soliton state.

In principle, if two centers are located close to each other in Hilbert space, then there could be trajectories oscillating around both of them simultaneously, thus spanning a larger phase space. Special initial conditions are required to excite them. An example of excitation of this type of trajectory is given in the next section.

\section{INPUT PULSES LAUNCHED INTO ONE CHANNEL}

In order to observe switching phenomena, the directional coupler is excited in only one channel [5]. Usually a sech-type function is considered the proper input in numerical simulations,

$$
\begin{aligned}
& U(\xi=0, \tau)=A_{0} \operatorname{sech}(\Omega \tau), \\
& V(\xi=0, \tau)=0,
\end{aligned}
$$

where $A_{0}$ is the pulse amplitude and $\Omega$ defines the width of the pulse (the width is inversely proportional to $\Omega$ ). The initial shape is not critical. We could have used Gaussian pulses and obtained similar results. The pulse shape mainly influences the amount of radiation produced. The output signal will consist of one or several soliton states of the coupler. The important initial parameter is the input energy. If the initial energy (after subtracting the part that will be radiated) is lower than that of the soliton state at the point $M$ of Fig. 1 , then we can suppose that the final result will be a symmetric soliton state plus perturbative oscillations around it. On the other hand if this energy is higher than the energy at the point $M$, then the final state would be one or several asymmetric $A$-type soliton states with perturbative oscillations around them. Hence, the energy of the states at the point $M\left(Q_{M}\right)$ can be considered as the threshold energy.

In reality, this simple behavior can be complicated by the presence of radiation and transient phenomena. As a result, the threshold energy is higher than $Q_{M}$ and the threshold itself is not sharp. We give here four examples of pulse evolution with initial energy close to the modified (taking into account the radiation) threshold. Figure 9(a) shows the distribution of the energy $\left(E_{1,2}\right)$ between both cores as a function of the propagation dis-
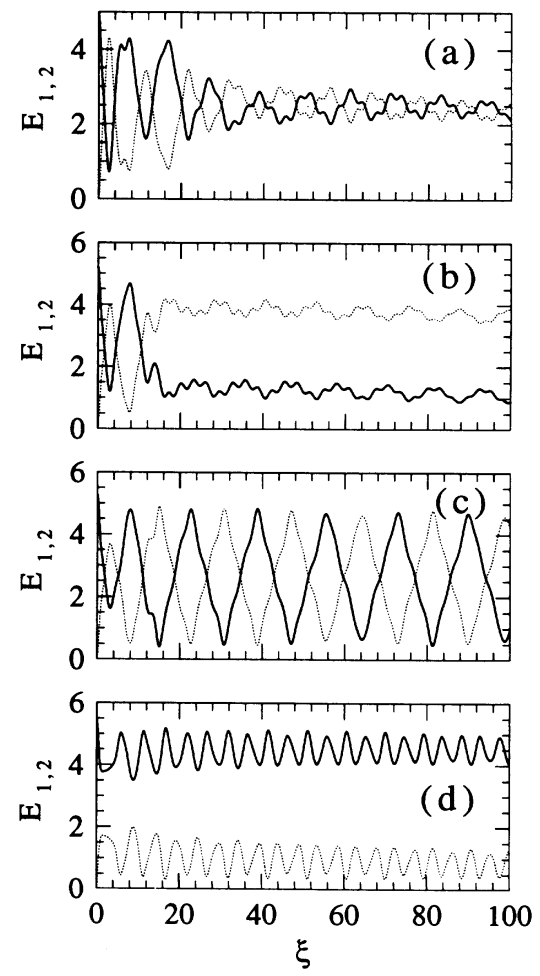

FIG. 9. The energy distribution along the coupler for the initial conditions given by Eq. (14). The corresponding parameters are $\Omega / K=2.1, K=1$, and (a) $A_{0}=2.300, Q / \sqrt{K}=5.03810$; (b) $A_{0}=2.337, Q / \sqrt{K}=5.20149 ;$ (c) $A_{0}=2.360, Q / \sqrt{K}$ $=5.30440$; (d) $A_{0}=2.400, Q / \sqrt{K}=5.48571$. 

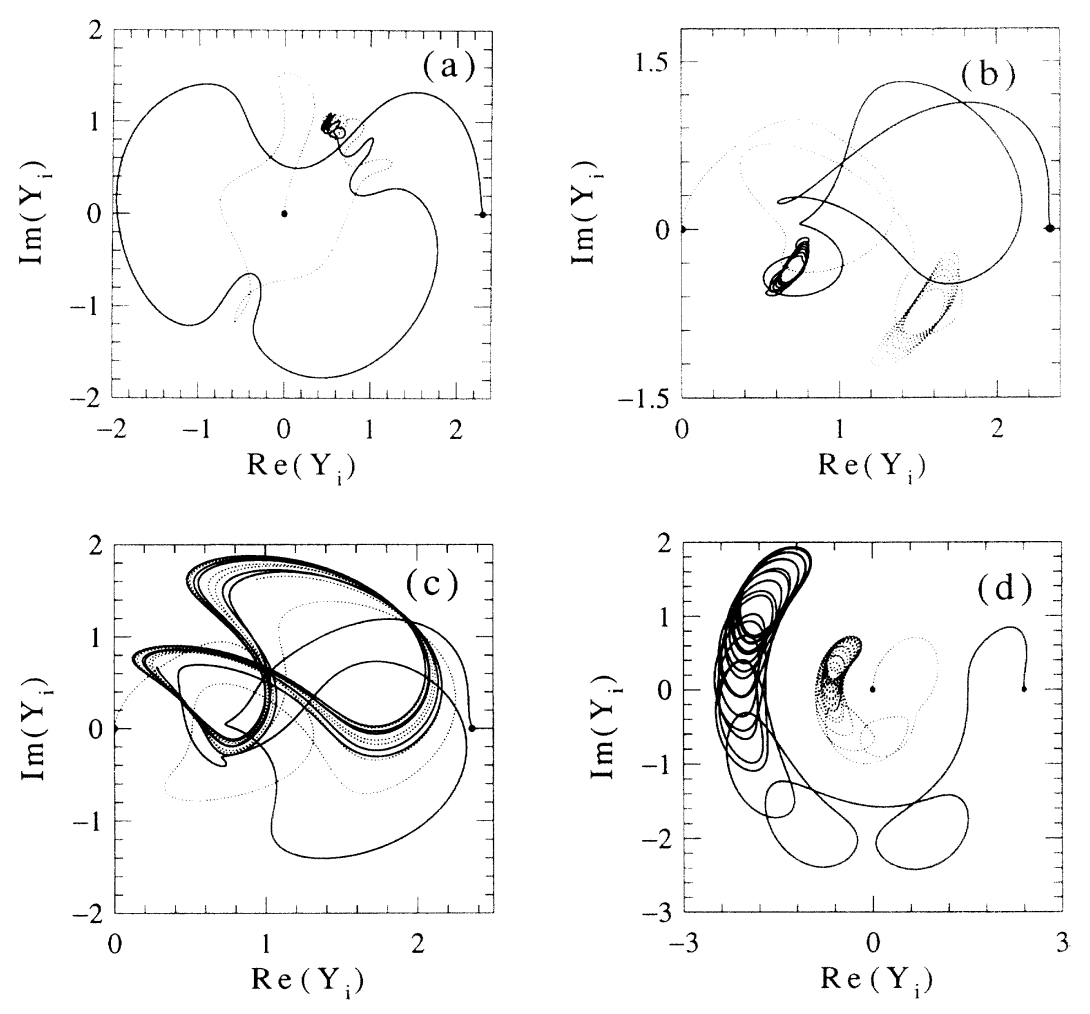

FIG. 10. Trajectories on the complex plane of the field amplitude at $\tau=0$ for the same cases as in Fig. 9.

tance $\xi$ for a directional coupler with $K=1$ and initial conditions given by Eq. (14), where $A_{0}=2.3$ and $\Omega=2$. 1 . This value of $\Omega$ is found to be the best to reduce radiation to the minimum value, and will be the same for all the cases exhibited in Fig. 8. The pulse energy in this case is $Q=5.04$. For comparison, the energy at the point $M$ is $Q_{M}=4.6$. The figure indicates that the solution oscillates around a symmetric soliton state with equal amplitudes. Note that there are a couple of large oscillations of the energy before the convergence to a symmetric state takes place. This convergence to the symmetric state is presented more explicitly using the trajectories on the complex plane [Fig. 10(a)]. After one rotation around the origin, when most of the radiation is emitted, both trajectories begin to rotate, with smaller amplitude, around the points corresponding to a stable symmetric soliton state. This transition is indicated in Fig. 11 by the left arrow.

This behavior is typical of initial conditions with low energies $(Q \leq 5)$. However, on decreasing the initial energy, the convergence to the symmetric soliton state becomes slower, and for very low amplitudes $\left(A_{0} \leq 1.5\right)$, when nonlinearity weakens, large oscillations around the symmetric state occur during the whole process, with the energy being transferred completely from one channel to the other during each oscillation. The period of the oscillations is $\pi /(2 K)$. This behavior is similar to that exhibited by a linear coupler excited by a continuous wave (cw). We recall that the pulses of the symmetric soliton state become wider in the limit $q \rightarrow K$. This is clearly seen from Eq. (10). Hence, this is the linear cw limit. Correspondingly, the widths of the pulses (initially short) increase upon each oscillation due to the dispersion. There is almost no radiation in this limit, in contrast to the cases with high energies.

If we increase slightly the energy of the input pulse, the behavior changes qualitatively. This is shown in Fig. 9(b), where $Q=5.20$. After an initial short stage with large amplitude oscillations $(0<\xi<12)$, the energy stays mainly in one channel with irregular small oscillations. The trajectory of the field at the origin on the complex plane corresponding to this process is shown in Fig. 10(b). Both trajectories gradually converge to the fixed points corresponding to the $A$-type soliton state close to the point with minimum energy in Fig. 1 (see also Fig. 11). Hence, this amount of energy $(Q=5.20)$ can be considered as a threshold.

It is feasible that further increase of energy would give qualitatively similar behavior. However, three singular

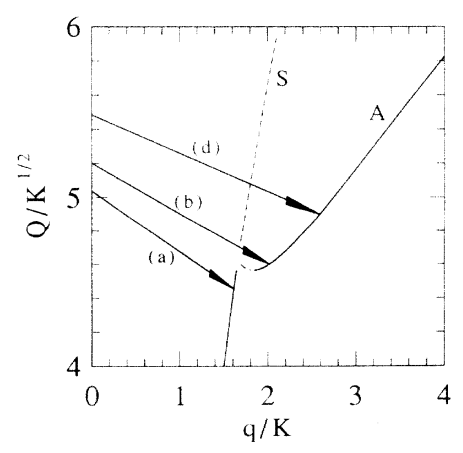

FIG. 11. Transformations shown in Figs. 9 and 10 on the $(Q, q)$ plane. The curves represent a part of Fig. 1 magnified for clarity. The arrows show the transformation upon propagation of the pulses given by the initial conditions (14) into specific soliton states. 
TABLE I. Generation of $A$-type soliton states.

\begin{tabular}{|c|c|c|c|c|c|}
\hline & $A_{0}$ & $\mathbf{\Omega}$ & $Q / \sqrt{K}$ & $N$ & $\frac{q}{K}$ values \\
\hline 1 & 2.8 & 2.1 & 7.46 & 1 & 5.804 \\
\hline 2 & 3.0 & 2.1 & 8.57 & 1 & 7.365 \\
\hline 3 & 3.5 & 2.1 & 11.67 & 1 & 11.825 \\
\hline 4 & 6.0 & 2.1 & 34.285 & 2 & 16 and 45 \\
\hline 5 & 4.0 & 1.0 & 32.0 & 2 & 20 and 20 \\
\hline 6 & 3.0 & 0.5 & 36.0 & 3 & 12,12 , and 8 \\
\hline 7 & 3.0 & 0.7 & 25.7 & 3 & $22,3.2$, and 3.2 \\
\hline
\end{tabular}

points (two stable and one unstable) are close together in phase space above the point of minimum energy. Due to this effect a small increase in energy $(Q=5.30)$ produces a trajectory which embraces both symmetric and antisymmetric states at the same time. Figures 9(c) and 10(c) clearly show that this is an example of an excitation of large oscillations around the two stable points mentioned in the previous section.

The above case is, of course, a very special one. When we increase the energy a little more, we directly excite an asymmetric $A$-type state with no long transition phenomena [Figs. 9(d) and 10(d)]. As usual, we have radiation and small nondecaying oscillations around the final state. The behavior is almost the same for larger input energies (14) until the input energy is high enough to excite two or more soliton states. Table I shows a few examples of numerical simulations, with different initial parameters $A_{0}$ and $\Omega$, confirming this behavior. In all of these examples the final states are one or more $A$-type asymmetric soliton states. The value $N$ in Table $I$ is the number of $A$ type soliton states. Note that the pulse with larger width (smaller $\Omega$ ) produces more soliton states. Their $q$ values are given in the last column. In some cases two soliton states with equal $q$ values are produced. In cases when two or more soliton states are produced, they propagate with different speeds. $K=1$ in all these latter cases.

\section{DISCUSSION}

From the above examples it is concluded that by using the approach of nonlinear dynamical systems generalized to systems with infinite degrees of freedom, it is possible to predict qualitatively the behavior of pulses propagating along a nonlinear directional coupler. Depending on the initial energy of the signal at the input, the output pulses are always close to one of the stable soliton states: a symmetric state below the point of bifurcation or an asymmetric $A$-state above the point of minimum energy. Although the total energy of the input signal is conserved, we can consider the central (signal) pulse as having energy losses related to the emission of radiation from the central pulse. Radiation plays an essential role in the processes of pulse transformations, allowing the pulses to adjust their energies to those corresponding to the stable soliton states.

Stable soliton states are always excited with small perturbations unless the initial condition is an exact soliton state. The perturbation behaves oscillatorily because its growth rate is purely imaginary for stable states. Hence, arbitrary initial conditions produce periodic motion around stable soliton states after the radiation is emitted. This general idea can be applied to any particular case.

For smooth initial pulses launched into one of two cores, there can be different regimes depending on the initial energy of the signal. (i) If the total energy of the input pulses is lower than some $Q_{0}\left(Q_{0}=Q_{M}+Q_{R}\right.$, where $Q_{M}$ is the energy at the point $M$ and $Q_{R} \approx 0.1 Q_{M}$ is the radiated energy), then the field will consist of radiation (which decreases the amount of energy in the signal) and periodic oscillations around a stable symmetric state. The oscillations gradually decay with propagation so that the signal converges to the symmetric soliton state. This convergence is slower for smaller initial energies. For very low initial energies, the oscillations have larger amplitudes and are nondecaying. In the linear limit, the coupler swaps the energy of the pulses between the channels as in the case of $\mathrm{cw}$ initial conditions. (ii) If the energy of the initial pulse is higher than $Q_{0}$, then the propagating field consists of radiation and periodic oscillations around an asymmetric $A$-type state. We can observe the unequal pulse amplitudes at the output of the coupler. There is an intermediate range of energies where symmetric and asymmetric states exist at the same value of energy. It can happen in this case that the motion consists of periodic oscillations around both symmetric and asymmetric soliton states simultaneously.

If the energy of the initial pulse is much higher than $Q_{0}$, so that it is enough to excite two or more soliton states, then several soliton states will appear. Each of these cases requires special consideration. In principle, these more complicated processes can be used for coding of information by solitons in transmission lines.

Another interesting possibility is to initially excite unstable soliton states and superimpose on them a certain small pulse, which acts as a perturbation to seed the instability. Two examples considered in this paper illustrate this possibility. Depending on the sign of the perturbation, the output of the nonlinear coupler will be one of the two different asymmetric $A$-type stable soliton states with the main part of the input energy concentrated in one of the channels. In this way, the energy of the output signal in each channel is controlled by a weak signal. Based on this idea new types of fast switches could be developed using nonlinear fiber couplers.

Finally, we would like to discuss the limits of applicability of the variational approach to this particular problem. Two recent papers [14-15] consider pulse propagation in nonlinear couplers using a variational approach with hyperbolic secant trial functions. This approach cannot be considered satisfactory in this problem for the following reasons. Using a trial function reduces the dynamical system with an infinite number of degrees of freedom to a dynamical system with a finite number of degrees of freedom. Solutions of the latter can greatly differ from the solutions of the former one. In particular:

(i) Using the trial function with fixed pulse shape [14] can "freeze" the second derivative on the left-hand side of the coupled set of equations (1). This is the reason why the authors of [14] obtained qualitatively, for pulse prop- 
agation, the same results as those given by Jensen [2] for continuous-wave propagation. The overall motion is periodic below and above the threshold and it converges to equal energy splitting at the threshold. This is not correct, as our numerical simulations show. The whole motion is more complicated than that obtained by using a simple trial function. The approximation of [14] roughly describes the field behavior of the half-beat-length coupler. However, even in this case it does not take into account the dependence of the output signal on the initial pulse shape (see, for example, [7]).

(ii) The prescribed evolution in pulse shapes in the variational approach does not follow the actual changes in shape given by the dynamical system defined by Eqs. (1). This explains why the structure of bifurcations and the stability properties of the soliton states found in [15] are in disagreement with the exact results of Refs. [29] and [30]. The saddle-type (unstable) points in Fig. 1(b) of [15] would correspond to the $A$-type asymmetric states in our notation. However, it is found in [15] that they exist only at medium energies and they are always unstable; this is in contradiction with our results. On the other hand, the central point in Figs. 1(b) and 1(c) of Ref. [15] (analog of the antisymmetric soliton states) is elliptic (i.e., stable). This also disagrees with the stability analysis of [30].

(iii) The variational approach does not take into account the radiative part of the field, but it is important, as we found from our numerical simulations. Emission of radiation from the signal can influence the evolution of the pulses during propagation. Moreover, radiation plays an important role, allowing arbitrary initial conditions to produce a particular soliton state.

(iv) The important parameter which defines the stability properties of the soliton states is the spatial frequency shift $q$, as we found in [30] and this work. This parameter is hidden when the variational approach is used [14-15]. This creates some additional difficulties in the analysis of pulse propagation and in the interpretation of the results.

This critical analysis shows that the variational approach can be applied to problems of pulse propagation generally only when the solution is already known from other methods. Roughly speaking, the use of trial functions is analogous to setting up rails in the complete space of functions defined by the dynamical system so that solutions can move only along those rails. Due to this constraint, the direction of motion may be erroneous in principle.

\section{CONCLUSIONS}

In conclusion, we have considered the propagation and transformation of short pulses in nonlinear directional couplers. We have interpreted the results of our numerical simulations in terms of Hamiltonian dynamical systems with an infinite number of degrees of freedom. In this approach, soliton states can be considered as singular points of the dynamical system. The singular points can be stable (elliptic type) or unstable (saddle type). We have shown that, for smooth initial functions which approach zero at infinity, the output signal at large distances consists of stable soliton states plus some amount of radiation. Any unstable soliton state is transformed, on propagation, into a stable soliton state and radiation.

\section{ACKNOWLEDGMENTS}

The work of N.A. is supported by the Australian Photonics Cooperative Research Centre (APCRC). The work of J.M.S.-C. was supported by the Comisión Interministerial de Ciencia y Tecnología under Contract No. TIC91-0361. One of the authors (N.A.) thanks Professor A.W. Snyder for useful discussions and Dr. A. Ankiewicz for a critical reading of our manuscript.
[1] A. A. Maier, Kvant. Electron. (Moscow) 9, 2296 (1982) [Sov. J. Quantum Electron. 12, 1490 (1982)].

[2] S. M. Jensen, IEEE J. Quantum Electron. QE-18, 1580 (1982).

[3] B. Daino, G. Gregory, and S. Wabnitz, J. Appl. Phys. 58, 4512 (1985).

[4] A. W. Snyder, D. J. Mitchell, L. Poladian, D. R. Rowland, and Y. Chen, J. Opt. Soc. Am. B 8, (1991).

[5] S. Trillo, S. Wabnitz, E. M. Wright, and G. I. Stegeman, Opt. Lett. 13, 672 (1988).

[6] E. M. Wright, G. I. Stegeman, and S. Wabnitz, Phys. Rev. A 40, 4455 (1989).

[7] G. D. Peng and A. Ankiewicz, Int. J. Nonlinear Opt. Phys. 1, 135 (1992).

[8] F. Kh. Abdullaev, R. M. Abrarov, and S. A. Darmanyan, Opt. Lett. 14, 131 (1989).

[9] A. Ankiewicz and G. D. Peng, Int. J. Optoelectron. 6, 15 (1991).

[10] F. Kh. Abdullaev and A. A. Abdumalikov, Pis'ma Zh. Tekh. Fiz. 14, 1041 (1988) [Sov. Tech. Phys. Lett. 14, 458
(1988)].

[11] J. M. Soto-Crespo and E. M. Wright, J. Appl. Phys. 70, 1365 (1989).

[12] C. Pare and M. Florjanczyk, Phys. Rev. A 41, 6287 (1990).

[13] Y. S. Kivshar and B. A. Malomed, Opt. Lett. 14, 1365 (1989).

[14] P. L. Chu, G. S. Peng, and B. A. Malomed, Opt. Lett. 18, 328 (1993).

[15] Y. S. Kivshar, Opt. Lett. 18, 7 (1993).

[16] M. Romagnoli, S. Trillo, and S. Wabnitz, Opt. Quantum Electron. 24, S1237 (1992).

[17] D. D. Gusovskii, E. M. Dianov, A. A. Maier, V. B. Neustruev, V. V. Osiko, A. M. Prokhorov, K. Yu. Sitarskii, and I. A. Scherbakov, Kvant. Electron. (Moscow) 14, 1144 (1987) [Sov. J. Quantum Electron. 17, 724 (1987)].

[18] A. A. Maier, Yu. N. Serdyuchenko, K. Yu. Sitarskii, M. Ya. Shchelev, and I. A. Scherbakov, Kvant. Electron. (Moscow) 14, 1157 (1987) [Sov. J. Quantum Electron. 17, 735 (1987)].

[19] S. F. Friberg, Y. Silberberg, M. K. Oliver, M. J. Andrejco, 
M. A. Saifi, and P. W. Smith, Appl. Phys. Lett. 51, 1135 (1987).

[20] S. F. Friberg, A. M. Weiner, Y. Silberberg, B. G. Sfez, and P. S. Smith, Opt. Lett. 13, 904 (1988).

[21] K. Kitayama and S. Wang, Appl. Phys. Lett. 43, 17 (1983).

[22] C. C. Yang, Opt. Lett. 16, 1641 (1991).

[23] A. A. Andronov and V. B. Khaikin, Theory of Oscillations, 3rd ed. (Nauka, Moscow, 1984).

[24] P. A. Cook, Nonlinear Dynamical Systems (Prentice-Hall, London, 1986).

[25] A. J. Lichtenberg and M. A. Lieberman, Regular and Chaotic Dynamics (Springer-Verlag, New York, 1992).
[26] C. R. Menyuk, J. Opt. Soc. Am. B 10, 1585 (1993).

[27] V. E. Zakharov and A. B. Shabat, Zh. Teor. Eksp. Fiz. 61, 118 (1971) [Sov. Phys. JETP 34, 62 (1972)].

[28] N. N. Akhmediev and J. M. Soto-Crespo, Phys. Rev. A 47, 1358 (1993).

[29] N. N. Akhmediev and A. Ankiewicz, Phys. Rev. Lett. 70, 2395 (1993).

[30] J. M. Soto-Crespo and N. N. Akhmediev, Phys. Rev. E 48, 4710 (1993).

[31] N. N. Akhmediev, Zh. Teor. Eksp. Fiz. 83, 545 (1982) [Sov. Phys. JETP 56, 299 (1982)]. 